\title{
Identifying factors associated with opioid cessation in a biracial sample using machine learning
}

Jiayi W. Cox ${ }^{1}$, Richard M. Sherva ${ }^{1}$, Kathryn L. Lunetta ${ }^{2}$ (, Richard Saitz $^{3}$, Mark Kon ${ }^{4}$, Henry R. Kranzler ${ }^{5}$, Joel Gelernter ${ }^{6,7}$ (D), Lindsay A. Farrer ${ }^{1,2,8 *}$ (1)

\begin{abstract}
${ }^{1}$ Department of Medicine (Biomedical Genetics), Boston University School of Medicine, Boston, MA 02118, USA
${ }^{2}$ Department of Biostatistics, Boston University School of Public Health, Boston, MA 02118, USA

${ }^{3}$ Department of Community Health Sciences, Boston University School of Public Health, Boston, MA 02118, USA

${ }^{4}$ Department of Mathematics and Statistics, Boston University College of Arts \& Sciences, Boston, MA 02215, USA

${ }^{5}$ Department of Psychiatry, Perelman School of Medicine, University of Pennsylvania and VISN 4 MIRECC, Crescenz VAMC,

Philadelphia, PA 19104, USA

${ }^{6}$ Departments of Psychiatry, Genetics and Neuroscience, Yale School of Medicine, New Haven, CT 06511, USA

${ }^{7}$ Department of Psychiatry, VA CT Healthcare Center, West Haven, CT 06516, USA

${ }^{8}$ Departments of Neurology, Ophthalmology and Epidemiology, Boston University Schools of Medicine and Public Health, Boston, MA 02118, USA
\end{abstract}

*Correspondence: Lindsay A. Farrer, Biomedical Genetics E200, Boston University School of Medicine, 72 East Concord Street, Boston, MA 02118, USA. farrer@bu.edu

Academic Editor: Hua Su, University of California, USA

Received: October 24, 2019 Accepted: December 18, 2019 Published: February 29, 2020

Cite this article: Cox JW, Sherva RM, Lunetta KL, Saitz R, Kon M, Kranzler HR, et al. Identifying factors associated with opioid cessation in a biracial sample using machine learning. Explor Med. 2020;1:27-41. https://doi.org/10.37349/ emed.2020.00003

\begin{abstract}
Aim: Racial disparities in opioid use disorder (OUD) management exist, however, and there is limited research on factors that influence opioid cessation in different population groups.

Methods: We employed multiple machine learning prediction algorithms least absolute shrinkage and selection operator, random forest, deep neural network, and support vector machine to assess factors associated with ceasing opioid use in a sample of 1,192 African Americans (AAs) and 2,557 individuals of European ancestry (EAs) who met Diagnostic and Statistical Manual of Mental Disorders, 5th Edition criteria for OUD. Values for nearly 4,000 variables reflecting demographics, alcohol and other drug use, general health, non-drug use behaviors, and diagnoses for other psychiatric disorders, were obtained for each participant from the SemiStructured Assessment for Drug Dependence and Alcoholism, a detailed semi-structured interview.

Results: Support vector machine models performed marginally better on average than other machine learning methods with maximum prediction accuracies of $75.4 \%$ in AAs and $79.4 \%$ in EAs. Subsequent stepwise regression considered the 83 most highly ranked variables across all methods and models and identified less recent cocaine use (AAs: odds ratio $(\mathrm{OR})=1.82, P=9.19 \times 10^{-5}$; EAs: OR $=1.91, P=3.30 \times 10^{-15}$ ), shorter duration of opioid use (AAs: $\mathrm{OR}=0.55, P=5.78 \times 10^{-6}$; EAs: $\mathrm{OR}=0.69, P=3.01 \times 10^{-7}$ ), and older age (AAs: $\mathrm{OR}=2.44, P=1.41 \times 10^{-12}$; EAs: $\left.\mathrm{OR}=2.00, P=5.74 \times 10^{-9}\right)$ as the strongest independent predictors of opioid cessation in both AAs and EAs. Attending self-help groups for OUD was also an independent predictor $(P<$


$0.05)$ in both population groups, while less gambling severity ( $\left.\mathrm{OR}=0.80, P=3.32 \times 10^{-2}\right)$ was specific to AAs and post-traumatic stress disorder recovery $\left(\mathrm{OR}=1.93, P=7.88 \times 10^{-5}\right)$, recent antisocial behaviors $(\mathrm{OR}=$ $\left.0.64, P=2.69 \times 10^{-3}\right)$, and atheism $\left(\mathrm{OR}=1.45, P=1.34 \times 10^{-2}\right)$ were specific to EAs. Factors related to drug use comprised about half of the significant independent predictors in both AAs and EAs, with other predictors related to non-drug use behaviors, psychiatric disorders, overall health, and demographics.

Conclusions: These proof-of-concept findings provide avenues for hypothesis-driven analysis, and will lead to further research on strategies to improve OUD management in EAs and AAs.

\section{Keywords}

Opioid use disorder, opioid cessation, machine learning, feature selection, outcome prediction

\section{Introduction}

Misuse of illicit and prescription opioids is a significant global problem that affects the health and economic welfare of individuals, families, and society. The U.S. opioid overdose rate has quadrupled since 1991 [1]. In 2017, more than 47,000 Americans died of an opioid overdose, and 36\% of these deaths involved prescription opioids [2]. A major goal in treating opioid use disorder (OUD) is abstinence, or complete cessation, of opioid use, other than the use of prescribed opioid replacement therapy. There is not a single, clinically accepted definition of cessation that specifies the length of abstinence required before an individual is no longer considered to have OUD [3, 4]. Diagnostic and Statistical Manual of Mental Disorders, 5th Edition (DSM5) considers sustained remission from OUD as a one-year period during which no criteria for the disorder (other than craving) are met [5].

Population differences affect multiple aspects of the current epidemic. Although opioid use nationally is higher among individuals of European ancestry (EAs) than African-Americans (AAs), the opioid death rate has increased more sharply among AAs than EAs [6]. AAs have less access to treatment for OUD [7], are less likely to obtain opioid prescriptions for pain management [8], and are incarcerated at a higher rate for illicit opioid use [9] than EAs. Previous research on OUD-related outcomes has been conducted primarily in combined ethnic groups or in EAs only [10], limiting the identification of key population differences in opioid use and treatment outcomes.

Although moderately correlated with opioid cessation, factors contributing to opioid treatment completion such as age, employment status, and age at first drug use have been identified from a mixed ethnicity sample [3]. Other factors are likely to influence cessation, such as pain experiences, general health, and the use of antidepressants [11-13]. Delineation of these factors could inform OUD treatment strategies that may differ across population groups; or could be useful for individuals with OUD who aim to reduce or stop their opioid use. However, studies thus far have tended to focus on a small number of clinically relevant factors such as the dosage, duration, and formulation of medication-assisted treatment of substance use disorders [14-16]. Large epidemiological studies of OUD [17-19] comprised of thousands of variables would allow a systemic, hypothesis-free query to identify factors predicting opioid cessation.

Statistical methods are generally limited in their ability to sort through large numbers of predictors [20]. Data mining using machine learning, which is particularly well suited for identifying predictive factors among thousands of variables [21, 22], has successfully identified predictor variables for a diverse set of outcomes [23-27]. Here, we applied multiple machine learning techniques to evaluate a large set of clinical, demographic, general health, and behavioral variables in a large, racially mixed cohort of individuals who were ascertained for cross-sectional genetic studies of substance use disorders, but not necessarily treated for OUD, to identify factors that are associated with opioid cessation (defined as self-reported last illicit opioid use and/or prescription opioids misuse $>1$ year before the interview date). Our study identified additional factors associated with cessation, including several that are population-specific. These findings support an individualized approach to improve the outcome of cessation attempts. 


\section{Materials and methods}

Participants and assessments

Participants for this study were selected from a cohort of 6,188 AAs and 6,835 EAs who were recruited for genetic studies of opioid, cocaine, or alcohol dependence between 2000 and 2017 through advertisements and treatment clinics at Yale University School of Medicine, the University of Connecticut Health Center, the University of Pennsylvania, the Medical University of South Carolina, and McLean Hospital [28, 29]. This cohort included affected sibling pairs and additional family members, as well as unrelated cases and controls. Probands with schizophrenia or bipolar affective disorder were excluded [28, 29]. Information about the use of various substances, demographics, general health, behavior, and other psychiatric illnesses was obtained by interview using the Semi-Structured Assessment for Drug Dependence and Alcoholism (SSADDA) [17, 30]. Substance use disorder (SUD) and psychiatric disorder diagnoses were established according to DSM-IV criteria. Institutional review boards from each recruitment site and Boston University (protocol \#H-26819) approved this study, and written informed consent was obtained from all participants.

\section{Opioid cessation definition}

Participants who were eligible for this analysis met at least two DSM-5 criteria for OUD, corresponding to a lifetime diagnosis of OUD. Current opioid cessation was determined by the response to the question, "When was the last time you used an opioid drug (including illicit methadone)." This question was asked as part of a series of items asked about illicit or non-prescribed use of opioids. Individuals who last used an opioid $>1$ year before the date of interview were considered to have achieved cessation and those whose last use of an opioid was $<6$ months before the interview date were classified as non-cessation. Persons who used opioids between 6 months and 1 year before the interview date were excluded from further analysis. Filtering steps yielding a sample of 1,192 AAs and 2,557 EAs for analysis are shown in Figure S1.

\section{Phenotype data processing}

Preprocessing of 3,956 SSADDA variables was performed prior to machine learning analyses. Variables with narrative or invariable responses, containing redundant information (e.g., specific date of different episodes, drug names), and with a response rate $<90 \%$ were removed. Missing values for binary and categorical variables were recoded as indicator variables to accommodate missing responses. Missing values for continuous variables were imputed to the population group mean value. Missing values for ordinal variables related to time since last drug use were assigned the highest level indicating less recent use. Z-score normalization (mean of 0 and variance of 1 ) was applied to continuous variables within each population to minimize scaling issues. The number of variables remaining after these steps was 3,315 in AAs and 3,738 in EAs.

\section{Machine learning analyses}

AAs and EAs were analyzed separately based on population differences in the epidemiology of opioid use and OUD. Variables were grouped into three nested sets defining three analytical models to explore the prediction accuracy blind to the individual's opioid or other drug use activities. This approach was adopted to enhance identification of non-drug use variables whose effects may be masked or confounded by variables related to drug use and are highly correlated with the cessation outcome. Model 1 contained all variables except those related to time since last opioid use that are strongly correlated with cessation (variable $n=3,093$ in AAs and $n=3,503$ in EAs). Model 2 further excluded all opioid-related variables ( $n=2,863$ in AAs and $n=$ 3,252 in EAs). Model 3 further excluded all drug use variables, leaving only demographic, non-SUD diagnoses and behaviors, and other health-related variables ( $n=1,656$ in AAs and $n=1,907$ in EAs). Models were evaluated using four machine learning methods described in Supplemental Materials to identify variables that are associated with opioid cessation. We modeled different types of inter-variable relationships between potential statistical predictors and the outcome using linear [least absolute shrinkage and selection operator, (LASSO) [31] and linear support vector machine (SVM) with recursive feature elimination (SVM) [32]] and non-linear [random forest (RF) with recursive feature elimination (RF) [33] and deep neural network (DNN) with feature selection (DNN) [34]] techniques. These four methods were applied to capture associated 
variables under different model assumptions and allow for different outcome-predictor relationships. Variables from each model that were associated with the highest accuracy reflected by either F1 score or area under the curve (AUC) and generated by each machine learning method were retained. The F1 score is a harmonic measure of precision [true positive / (true positive + false negative)] and recall [true positive / (true positive + true negative)], defined by $2 \times$ (precision $\times$ recall) / (precision + recall) at a given case/ control split, and AUC is an overall evaluation of model performance that accounts for the true positive and false positive rates for all possible diagnostic splits $[35,36]$. Both measurements were considered because of their popularity in clinical settings [37]. The F1 score was used to assess accuracy due to limitations of the AUC, which includes bias when performed on imbalanced datasets as well as impractical and uninterpretable split points for evaluation $[35,38]$.

\section{Statistical methods for testing the association of opioid cessation with phenotypic variables}

To determine which variables selected by the machine learning methods are independently associated with cessation, we applied different cutoffs for the importance measurement of each machine learning method: namely the odds ratio (OR) for LASSO, coefficient [39] denoted by weight for SVM, feature importance [39] for $\mathrm{RF}$, and activation potential [34] for DNN. For LASSO, we chose variables that yielded ORs $>1.05$ or $<0.95$. We applied the following criteria for selecting variables from SVM and RF analyses depending on the number of variables ( $n$ ) selected for each model: (1) if $n>200$, the top 30\% of variables measured by absolute weight in SVM or importance in RF were designated as high impact, (2) if $100<n<200$, the top $50 \%$ were selected, and (3) if $n<100$, all variables were designated as high impact. For DNN, all selected variables were designated "high impact". Joint association tests were performed using bi-directional stepwise logistic regression that included 83 "high-impact" variables culled from three models across four machine learning methods in the AA and EA datasets. Variables that yielded the highest Akaike information criterion (AIC) with $P<0.05$ from bi-directional stepwise logistic regression were grouped into "drug related", "behavioral", "other health", and "demographic" categories.

\section{Results}

Characteristics of the study samples are shown in Table 1. The sample included 1,069 unrelated AAs and 2,252 unrelated EAs, and $123 \mathrm{AA}$ and 305 EA participants who were members of families containing a pair of siblings concordant for opioid or cocaine dependence. There is a higher proportion of females among individuals who ceased opioid use in both AAs ( $\left.\mathrm{OR}=1.35, P=6.7 \times 10^{-3}\right)$ and EAs (OR $\left.=1.31, P=1.1 \times 10^{-3}\right)$ compared to those who did not cease. Participants who ceased opioid use were also older by an average of 3.18 years in the AA group $\left(P=1.0 \times 10^{-10}\right)$ and 6.1 years in the EA group $\left(P=2.2 \times 10^{-16}\right)$ than those who did not cease use. The mean number of lifetime DSM-5 OUD criteria did not significantly differentiate individuals who ceased opioid use from those who did not.

Table 1. Participant characteristics

\begin{tabular}{llll}
\hline & & \multicolumn{2}{c}{ Time since last use } \\
& & $\leq 6$ month (not cease) & $>$ 1 year (ceased) \\
\hline AAs & Number (\% female) & $701(33.5 \%)$ & $491(40.5 \%)$ \\
& Age (Mean \pm SD) & $42.6 \pm 8.5$ & $45.6 \pm 8.3$ \\
& OUD Symptom Counts (Mean \pm SD) & $7.8 \pm 2.4$ & $7.6 \pm 2.5$ \\
EAs & Number of families (number in families) & $35(76)$ & $23(47)$ \\
$(N=2557)$ & Number (\% female) & $1714(34.4 \%)$ & $843(40.6 \%)$ \\
& Age (Mean \pm SD) & $34.4 \pm 10$ & $40.5 \pm 10.3$ \\
& OuD symptom Counts (Mean \pm SD) & $8.8 \pm 1.9$ & $8.4 \pm 2.3$ \\
& Number of families (number in families) & $114(241)$ & $31(64)$ \\
\hline
\end{tabular}

SD: standard deviation 


\section{Feature selection}

The F1 score was generally higher across models in both AAs and EAs using SVM than the other machine learning algorithms (Figure S2), although the differences in F1 score across methods were generally small, especially for models 1 and 2. A detailed discussion of the performance of each method for the three models is provided in Supplemental Materials and Figure S2.

Figure 1 shows the overlap of high impact variables chosen by the four machine learning methods. LASSO "high impact" variables almost entirely overlap with those from the other methods, while DNN-selected variables overlap the least with other method-selected variables. The majority of variables selected by nonLASSO methods are unique to those methods, however, there was high overlap in "high impact" variables selected by SVM and RF. Age was among the five top-ranked variables consistently identified by each method for each model in both AAs and EAs (Table S1). Time since last cocaine use (injection) and recent cocaine use symptoms were selected by all machine learning methods for models 1 and 2 for both AAs and EAs. Also in model 1 analysis, age at first heavy opioid use and years of heroin use were identified in AAs by all machine learning methods. Several variables were selected by all methods in model 2 analyses including time since last cocaine use (injection) in both populations, time since first tobacco use in AAs, and body mass index, age at heaviest weight, and age started heavy cocaine use in EAs. Specific to model 3, positive HIV status, number of children, number of months employed in the last year, and jobless while having drinking and drug problems were selected by all methods in AAs. Under the same model, age at the heaviest weight, co-morbid illnesses, and time since exhibiting last antisocial behavior were selected by all methods in EAs.

A.

(1)

(2)

(3)

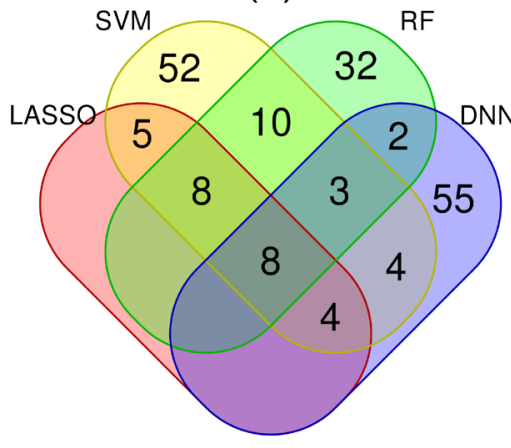

B.

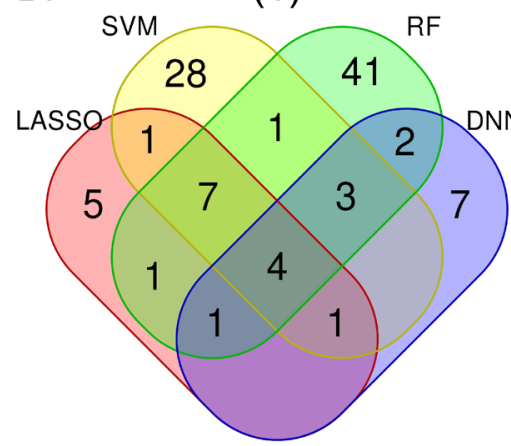

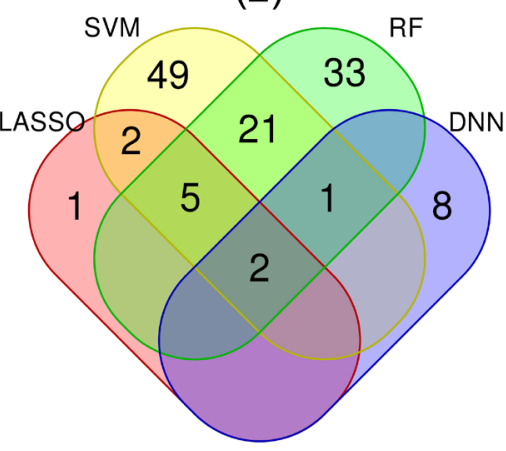

(2)

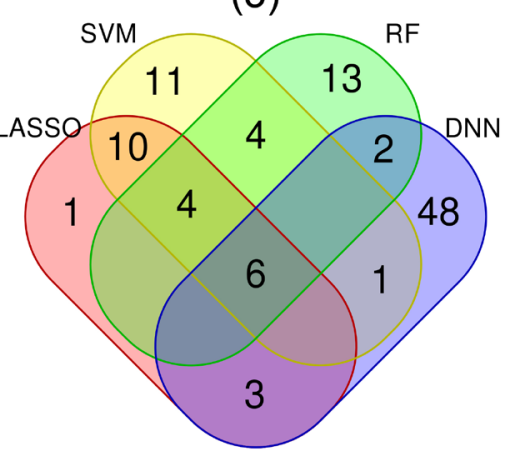

(3)

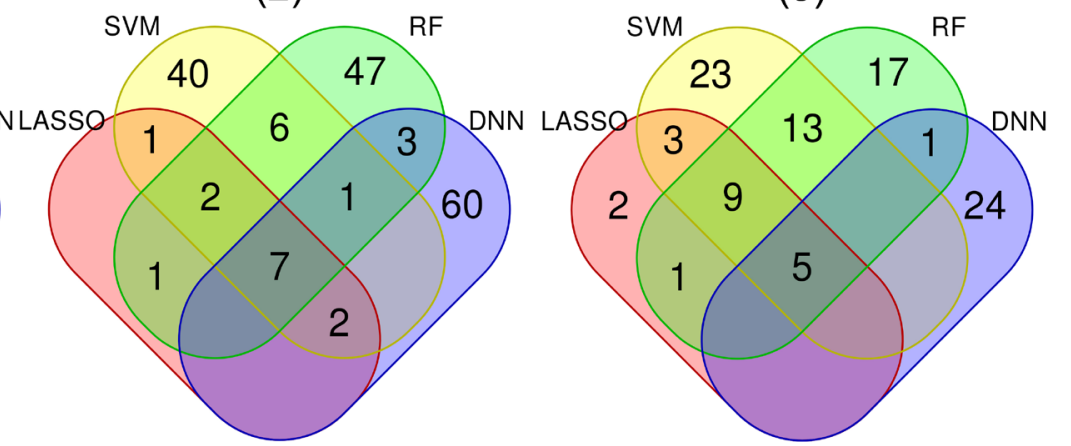

Figure 1. Number of overlapping "high impact" variables selected by each machine learning method based on the importance measurement in African Americans (A) and European Americans (B) for models (1), (2), and (3). Model 1 includes all variables except for those that are confounded with opioid cessation. Model 2 includes all variables in Model 1 except opioid-related variables. Model 3 includes all variables in Model 2 except drug use-related variables. Colors represent particular machine learning methods: pink = LASSO, light yellow $=$ SVM, light green $=$ RF, light blue $=$ DNN

\section{Factors associated with opioid cessation}

Stepwise regression analysis that considered 83 "high impact" variables culled from all models and machine learning methods (Table 2, Table S2) identified both population specific factors and non-population specific factors associated with opioid cessation. Variables related to drug use comprised over $50 \%$ of the nominally significant predictors of opioid cessation in AAs (29 of 41) and EAs (27 of 50). 
Drug related variables were among the most significant positively associated predictors of opioid cessation in AAs and EAs including time since last cocaine injection $\left(\mathrm{OR}_{\mathrm{AAs}}=2.30\right.$ per level change, $P_{\mathrm{AAs}}=9.11$ $\left.\times 10^{-6}\right)$ or use $\left(\mathrm{OR}_{\mathrm{EAs}}=1.91\right.$ per level change, $\left.P_{\mathrm{EAs}}=3.30 \times 10^{-15}\right)$, while more years using heroin $\left(\mathrm{OR}_{\mathrm{AAs}}=0.55\right.$ per standard deviation (SD) change, $\left.P_{\mathrm{AAs}}=5.78 \times 10^{-6}\right)$ or being older at first heavy opioid use $\left(\mathrm{OR}_{\mathrm{EAs}}=0.56\right.$ per SD change, $P_{\text {EAs }}=2.67 \times 10^{-12}$ ) decreased the odds of the outcome. Several other drug use variables were associated with greater odds of cessation including "more time since last had alcohol symptoms lasting $>1$ month" $\left(\mathrm{OR}_{\mathrm{AAs}}=1.45\right.$ per level change, $\left.P_{\mathrm{AAs}}=2.84 \times 10^{-3}\right)\left(\mathrm{OR}_{\mathrm{EAs}}=1.34\right.$ per level change, $\left.P_{\mathrm{EAs}}=4.13 \times 10^{-5}\right)$, "had 2 marijuana symptoms lasting $>1$ month" $\left(\mathrm{OR}_{\mathrm{AAs}}=2.13, P_{\mathrm{AAs}}=4.83 \times 10^{-3}\right)$ or "marijuana interfered with work or home activities" $\left(\mathrm{OR}_{\mathrm{EA}}=1.67, P_{\mathrm{EA}}=1.61 \times 10^{-3}\right)$, "smoked less frequently after waking up" $\left(\mathrm{OR}_{\mathrm{AAs}}=1.75\right.$, $P_{\text {AAs }}=7.76 \times 10^{-3}$ ) or the Fagerstrom Test for Nicotine Dependence (FTND) item "able to cut down smoking" $\left(\mathrm{OR}_{\mathrm{EAs}}=1.28, P_{\mathrm{EAs}}=3.69 \times 10^{-2}\right)$. Having attended a self-help group for OUD $\left(\mathrm{OR}_{\mathrm{AAs}}=1.72, P_{\mathrm{AAs}}=1.41 \times 10^{-2}\right)$ or started attendance at an OUD self-help group sooner $\left(\mathrm{OR}_{\mathrm{EAS}}=1.28\right.$ per level change, $\left.P_{\mathrm{EAs}}=2.4 \times 10^{-3}\right)$ also increased the odds of cessation.

Several variables related to other mental health issues were also associated with opioid cessation. Selfharm $\left(\mathrm{OR}_{\mathrm{AAs}}=1.39, P_{\mathrm{AAs}}=1.96 \times 10^{-2}\right)$ or suicidal ideation $\left(\mathrm{OR}_{\mathrm{EAs}}=1.2, P_{\mathrm{EAs}}=8.08 \times 10^{-3}\right)$ were associated with significantly higher odds of cessation. Prior history of a depressive episode lasting $>1$ week $\left(\mathrm{OR}_{\mathrm{AAs}}=1.31\right.$, $\left.P_{\mathrm{AAs}}=1.66 \times 10^{-2}\right)$ or having drug-use associated depression $\left(\mathrm{OR}_{\mathrm{EAs}}=1.64, P_{\mathrm{EAs}}=2.55 \times 10^{-3}\right)$ predicted higher odds of cessation. Pathological gambling severity $\left(\mathrm{OR}_{\mathrm{AAs}}=0.8, P_{\mathrm{AAs}}=3.32 \times 10^{-2}\right)$ and no anxiety for longer than six months $\left(\mathrm{OR}_{\mathrm{AAs}}=1.72, P_{\mathrm{AAs}}=2.06 \times 10^{-3}\right)$ were significantly associated with cessation in AAs. In EAs, recovering from an event causing post-traumatic stress disorder (PTSD) assessed by the question "no fear in most disturbing/traumatizing event" $\left(\mathrm{OR}_{\mathrm{EAs}}=1.93, P_{\mathrm{EAs}}=1.66 \times 10^{-6}\right)$, less recent antisocial behavior episodes $\left(\mathrm{OR}_{\mathrm{EAs}}=1.35\right.$ per SD change in age, $\left.P_{\mathrm{EAs}}=1.03 \times 10^{-4}\right)$, and unsafely raced cars $\left(\mathrm{OR}_{\mathrm{EAs}}=1.78, P_{\mathrm{EAs}}=3.79 \times 10^{-3}\right)$ were associated with increased odds of cessation.

Table 2. Variables associated with opioid cessation at $P<0.01$ in the (A) African American and (B) European ancestry groups

\begin{tabular}{|c|c|c|c|}
\hline \multicolumn{4}{|c|}{ (A) African American } \\
\hline & Variable & OR & $P$-value \\
\hline \multirow[t]{17}{*}{ Drug related } & Time since 1 st opioid treatment $^{*}$ & 1.56 & 1.90E-04 \\
\hline & Older age at first opioid symptoms $\$$ & 0.46 & 2.23E-05 \\
\hline & Number of years using heroin $\$$ & 0.55 & $5.78 \mathrm{E}-06$ \\
\hline & Depressed after reducing cocaine use & 0.53 & 4.05E-03 \\
\hline & Time since last injected cocaine ${ }^{*}$ & 2.30 & $9.11 \mathrm{E}-06$ \\
\hline & Time since last used cocaine ${ }^{*}$ & 1.82 & $9.19 \mathrm{E}-05$ \\
\hline & Time since last stayed high in cocaine ${ }^{*}$ & 1.41 & 2.93E-03 \\
\hline & Used cocaine $<11$ times within year of interview & 2.67 & $1.38 \mathrm{E}-03$ \\
\hline & Treated in outpatient program for cocaine use & 1.88 & 4.06E-03 \\
\hline & Time since of first cocaine craving ${ }^{*}$ & 0.71 & 1.59E-03 \\
\hline & Never injected cocaine & 2.53 & 1.75E-03 \\
\hline & Often used marijuana more than intended to & 0.40 & 5.67E-04 \\
\hline & Mixed alcohol and drugs $>3$ times in 12 months & 0.51 & $2.08 \mathrm{E}-03$ \\
\hline & Time since last had alcohol symptoms lasting $>1$ month $^{*}$ & 1.45 & $2.84 \mathrm{E}-03$ \\
\hline & Smoked less frequently after waking up & 1.75 & 7.76E-03 \\
\hline & Older age at first cigarette ${ }^{\$}$ & 1.31 & $6.24 \mathrm{E}-03$ \\
\hline & Had 2 marijuana symptoms lasting a month & 2.13 & 4.83E-03 \\
\hline \multirow[t]{2}{*}{ Other Health } & HIV positive & 2.47 & 1.39E-03 \\
\hline & Health has always been better than now & 0.62 & $9.64 \mathrm{E}-03$ \\
\hline \multirow[t]{2}{*}{ Demographic } & Female sex & 1.91 & 1.83E-03 \\
\hline & Current age & 2.44 & $1.41 \mathrm{E}-12$ \\
\hline
\end{tabular}




\begin{tabular}{|c|c|c|c|}
\hline & Variable & OR & $P$-value \\
\hline \multirow[t]{19}{*}{ Drug related } & 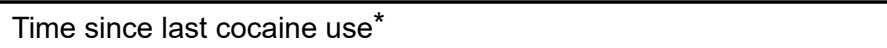 & 1.91 & $3.30 \mathrm{E}-15$ \\
\hline & Older age at first heavy opioid use ${ }^{\$}$ & 0.56 & 2.67E-12 \\
\hline & Number of years using heroin $\$$ & 0.69 & 3.01E-07 \\
\hline & Time since last cocaine injection* & 1.85 & $2.38 \mathrm{E}-06$ \\
\hline & Time since last had alcohol symptoms that last $>1$ month $^{*}$ & 1.34 & 4.13E-05 \\
\hline & $>20$ outpatient visits for drug/psychiatric problems in the last year & 1.76 & $9.56 \mathrm{E}-06$ \\
\hline & Time since opioid treatment initiation ${ }^{*}$ & 1.53 & $2.24 \mathrm{E}-06$ \\
\hline & Used cocaine $>11$ times in last year & 0.47 & 1.09E-05 \\
\hline & Time since first used opioid $1 /$ week for $>1$ month $^{*}$ & 1.41 & $1.10 \mathrm{E}-04$ \\
\hline & Have injected cocaine & 2.01 & 1.66E-04 \\
\hline & Older age at first heavy cocaine use ${ }^{\$}$ & 1.27 & 7.40E-04 \\
\hline & Marijuana interfered with work/home & 1.67 & $1.61 \mathrm{E}-03$ \\
\hline & Time since one started opioid self-help group* & 1.28 & $2.40 \mathrm{E}-03$ \\
\hline & Time since last feel high on cocaine for $>1$ day $^{*}$ & 1.33 & 1.15E-03 \\
\hline & Time since last attended cocaine self-help group* & 0.76 & $3.27 \mathrm{E}-04$ \\
\hline & Used tobacco but not addicted & 0.60 & $3.72 \mathrm{E}-03$ \\
\hline & Disclosed problems with cocaine usage to professional & 1.65 & $2.45 \mathrm{E}-03$ \\
\hline & Stopped using stimulants for $>3$ month & 1.99 & $2.38 \mathrm{E}-03$ \\
\hline & Drinking resulted in objections or problems with family and work & 1.48 & 4.39E-03 \\
\hline \multirow[t]{13}{*}{ Behavioral } & $<3$ ASP criteria in 12-month period & 0.64 & 2.69E-03 \\
\hline & Often failed to pay debts & 0.68 & 2.17E-03 \\
\hline & Suspended or expelled from school & 0.67 & 2.16E-03 \\
\hline & Time since last had suicidal ideation ${ }^{*}$ & 1.20 & $8.08 \mathrm{E}-03$ \\
\hline & Less recent since last had antisocial behaviors $\$$ & 1.35 & 1.03E-04 \\
\hline & No fear of most disturbing/traumatizing event & 1.93 & 1.66E-06 \\
\hline & Avoided scenes that reminded of traumatic event & 1.88 & $7.88 \mathrm{E}-05$ \\
\hline & Had OCD behaviors when depressed & 0.49 & 3.23E-04 \\
\hline & Feeling distracted & 1.56 & 8.15E-04 \\
\hline & Unsafely raced cars & 0.56 & 3.79E-03 \\
\hline & Depression always started with drug problems & 1.64 & $2.55 \mathrm{E}-03$ \\
\hline & Number of depression symptoms & 1.46 & 8.99E-03 \\
\hline & Have outstanding emotional problem & 1.63 & $5.55 \mathrm{E}-03$ \\
\hline Other Health & Body mass index & 1.32 & $3.59 \mathrm{E}-06$ \\
\hline \multirow[t]{2}{*}{ Demographics } & Household income & 1.15 & $1.30 \mathrm{E}-03$ \\
\hline & Current age & 2.00 & 5.74E-09 \\
\hline
\end{tabular}

OR = odds ratio. ${ }^{*}$ Categorical variable: 1 = within the last two weeks, $2=$ two weeks to less than one month ago, $3=$ one month to less than six months ago, $4=$ six months to one year ago, $5=$ more than a year ago. OR represents the factor increase per level change. ${ }^{\$}$ Continuous variable: OR represents the factor increase per standard deviation unit

Older age was one of the most significantly associated variables with opioid cessation in both population groups $\left(\mathrm{OR}_{\mathrm{AAs}}=2.44\right.$ per SD change, $P_{\mathrm{AAs}}=1.41 \times 10^{-12}, \mathrm{OR}_{\mathrm{EAs}}=2.00$ per SD change, $\left.P_{\mathrm{EAs}}=5.74 \times 10^{-9}\right) . \mathrm{In}$ AAs, female sex $\left(\mathrm{OR}_{\mathrm{AAs}}=1.91, P_{\mathrm{AAs}}=1.83 \times 10^{-3}\right)$ and fulltime employment $\left(\mathrm{OR}_{\mathrm{AAs}}=1.84, P_{\mathrm{AAs}}=1.82 \times 10^{-2}\right)$ were associated with a greater likelihood of opioid cessation, while having been raised primarily by a single parent $\left(\mathrm{OR}_{\mathrm{AAs}}=0.63, P_{\mathrm{AAs}}=1.3 \times 10^{-2}\right)$ was associated with not achieving cessation. Other variables that were significantly associated with opioid cessation in AAs included HIV positive status (OR $\left.=2.47, P=1.39 \times 10^{-3}\right)$, whereas in EAs higher body mass index (OR $=1.32$ per SD change, $\left.P=2.58 \times 10^{-6}\right)$, have asthma $(\mathrm{OR}=0.68, P$ 
$\left.=1.22 \times 10^{-2}\right)$, higher household income $\left(\mathrm{OR}=1.15, P=1.3 \times 10^{-3}\right)$, and being an atheist $(\mathrm{OR}=1.45, P=1.34 \times$ $10^{-2}$ ) were significantly associated with opioid cessation.

\section{Discussion}

We employed both regression and non-regression-based machine learning approaches to evaluate the association of more than 3,000 variables related to SUDs and other psychiatric disorders, other health-related behaviors, and demographic variables with opioid cessation among EAs and AAs assessed in a cross-sectional study of opioid, cocaine, and/or alcohol dependence. We observed moderate-to-high predictive accuracy across all methods; SVM, on average, marginally outperformed the other methods. Although the specific set of associated variables differed in EAs and AAs, a common profile emerged. People who ceased opioid use tended to be older, initiated drug use later in life, had used opioids for a shorter period, experienced fewer problems related to cocaine or alcohol use, were currently employed, and had recovered from other psychiatric disorders including depression and PTSD compared to those whose opioid use persisted.

Previous research using machine learning for addiction outcomes focused mainly on predictive accuracy, although a few studies attempted to identify and interpret specific variables that were associated with the outcomes [3, 4, 40, 41]. Acion et al.[3] reported that ensemble super learning was superior to other machine learning methods, and used penalized regression, SVM, and neural networks for predicting SUD treatment success indicated by treatment discharge status in a Hispanic cohort. In that study, less than $10 \%$ of participants had problems with cocaine or illicit opioids and fewer than 30 variables were assessed. In contrast, we evaluated several thousand variables, including detailed measures of drug-use activities and psychiatric disorders, and ranked the importance of the top-ranked variables with four distinct machine learning algorithms. Gowin et al.[40] identified regional brain activity changes predicting relapse from imaging data on fewer than 70 methamphetamine-dependent patients without including any lifestyle factors. Che et al.[4] applied deep learning to electronic health record data to identify people with short-term or long-term opioid use or dependence. Similar to our study, they identified associations with comorbid substance use and anxiety disorders [4]. Several other studies used only regression-based methods to identify variables associated with opioid and stimulant dependence [42], cocaine dependence [43] and alcohol dependence [44], which might not capture other relationships among variables. Several of the non-regression-based methods we employed have also been applied in other studies, which focused mainly on brain magnetic resonance imaging (MRI) traits as predictors of substance use disorder diagnoses [24, 25, 27, 40].

We identified association of opioid cessation with several variables that were previously associated with OUD or OUD-related conditions including co-morbid drug use, antisocial behavior, suicidal thoughts, HIV infection, and asthma [45-50]. Our finding that the majority of people who ceased opioids (60\% in AAs and $66 \%$ in EAs) also ceased cocaine use is consistent with evidence of high rates of co-occurring OUD and cocaine use disorder (CUD) [51, 52]. This finding also supports the use of treatment strategies that target both disorders $[51,53]$ and suggests that ceasing use of one substance might influence the ability to cease use of the other. Alternatively, ceasing both opioid and cocaine use may reflect self-selection for inclusion in our genetic studies in which $43 \%$ of AA and 32\% of EA participants were ascertained for CUD. Our findings are also consistent with observations that a failure to address tobacco use lowers the efficacy of opioid cessation treatment [54] and that a behavioral intervention in patients with antisocial personality disorder reduces substance use [55]. Unlike problems that are associated with other drug use and lower odds of opioid cessation, we found that cannabis use-related problems (e.g., two marijuana symptoms lasting a month, marijuana interfering with work) are associated with higher odds of opioid cessation. This finding is puzzling and not immediately explainable. Although our observation that cannabis users had better success quitting opioids is consistent with prior reports of association of cannabis use and reduced opioid withdrawal symptoms and pain $[56,57]$, recently we showed that cannabis as a replacement for opioids as treatment strategy for OUD could be harmful [58]. Previous findings of the co-occurrence of drug addiction, suicide attempts, depression, family conflicts, and PTSD, which may suggest bi-directional casual relationships [45-50], are consistent with 
our observation that better management of comorbid psychiatric problems (fewer recent suicide attempts and psychiatric symptoms) increases the likelihood of opioid cessation or vice versa.

Acion et al.[3] and we identified age, employment status, and age at first drug use as factors for treatment success. The protective effect of older age may be due to ascertainment bias because persons who survived severe dependence are more likely to have stopped using opioids. Full-time employment likely reduces the time or urge for persons dependent on opioids to seek and use the drug. In addition, drug screening associated with some jobs may reduce the likelihood of current opioid use [59]. Quitting opioids also make it easier to find/maintain a job. The inverse correlation of age at first drug use and opioid cessation may reflect the increased difficulty of reversing the effect of long-term opioid exposure on the brain reward system [60] or increased severity associated with earlier onset.

Several variables that were significantly associated with opioid cessation related to non-substancerelated behavior were population specific. Although these findings may be due in part to differences between AAs and EAs in willingness to endorse these behaviors, previous studies showed that AAs were more likely than EAs to report prolonged gambling and problems associated with gambling [61, 62]. One explanation for our findings of significant associations of ceasing opioids with a self-reported HIV diagnosis in AAs is that OUD patients with severe or life-threatening illnesses are more likely to seek or adhere to treatment [63], an idea supported by evidence that HIV-infected patients have better treatment outcomes for OUD [64-66]. Alternatively, poorer general health may lead to reduced drug use [67] (the so-called "sick quitter"). In contrast, antisocial behavior, recovery from PTSD, and being an atheist were associated with opioid cessation in EAs only. Prior research may provide insight into these EA-specific patterns. One study reported antisocial behaviors in EA children were significantly associated with substance initiation while the association was less strong in AA children [68], although the impact on opioid use was not assessed. PTSD and being an atheist identified in EAs might be due to the racial difference in exposure to traumatic events and belief diversities $[69,70]$. Previous evidence about the effect of religion on SUDs is contradictory. One study showed that loss of religiosity between childhood and adulthood was associated with increased substance use while recent religiosity increased the odds of illicit drug use in the past year [71]. Alternatively, the smaller sample of AAs might have limited our ability to detect these associations in that group.

The current study has several strengths. First, because the input dataset contains thousands of variables related to drug use activities, psychiatric disorders, medical history, and demographics obtained from several thousand individuals meeting DSM-5 criteria for OUD, we were able to explore many factors in addition to those included in other studies. Second, both linear and non-linear machine learning methods were employed to model the true underlying relationship between the variables and outcome, which increased the number of factors we identified. Third, we evaluated three models for each machine learning method in order to better understand the contribution of opioid and other drug use information. Fourth, we considered only independent variables in the association analyses to prevent over-representation of correlated factors. Finally, although there is no published "gold standard" predictive model against which to compare our results, the $80 \%$ predictive accuracy we achieved is similar to that seen in other machine learning studies $[3,4,40,41]$.

Limitations of this work should be noted. First, given the cross-sectional nature of our data and the over $90 \%$ relapse rate for OUD [72], many individuals classified as not using opioids may have subsequently relapsed to opioid use. However, it has been shown that prior abstinence is predictive of future abstinence, therefore people who ceased opioids are more likely to cease again even when relapse occurs [73]. Second, the machine learning analyses were based on samples that may have been underpowered to detect associations with some variables compared to other studies that included tens of thousands of individuals [74]. Third, most persons in our cohort were evaluated prior to the current opioid epidemic and may not reflect recent secular trends in the prevalence and associated features of OUD. Fourth, associations of cessation with some variables and overall prediction accuracy may have been inflated because our analysis did not fully account for familial correlations. Fifth, in spite of the large number of variables that were included in the machine learning analyses, potentially important variables such as the reasons for first use and details of treatment and support programs were unavailable. However, we identified attending an opioid addiction self-help group 
as associated with successful cessation, which is consistent with the reported benefit of self-help groups [75]. Sixth, the rate of response to many interview questions was substantially higher in EAs, while the sample size was twice that of AAs, which could account for some of the observed racial differences in predictive models. Related to this concern, our use of mean imputation for missing data may have been overly conservative. Other methods, including cold/hot deck (such as K nearest neighbors) and multiple imputation [76], may have provided additional information but were not appropriate for this dataset given its size, use of continuous, ordinal, and binary variable coding, non-linear relationships among variables, and lack of an appropriate external reference. Seventh, given the limited amount of temporal information with respect to many of the potential risk factors for opioid cessation in this cross-sectional sample, it is difficult to determine the effect direction for many of the observed associations. Because of these limitations, our findings require external validation in larger samples before they can be incorporated in prediction models for clinical purposes. Finally, while some of the factors we identified are plausible and consistent with prior studies, other factors such as atheism are not immediately interpretable. Thus, because our research is atheoretical, results should be interpreted with caution and be validated before implemented in clinical practice.

In conclusion, we analyzed a large number of variables including demographic, behavioral, health and drug use activities using machine learning techniques with feature selection and found variables in a wide range of domains that were associated with cessation. These included some that are consistent with prior literature, plausible but have not been well studied, and do not have readily apparent explanations for their associations. Our findings suggest hypotheses for future studies and could inform how one might increase the likelihood of cessation with and without treatment. These results also support several widely known treatment strategies for OUD, such as treating psychiatric comorbidity, adding wraparound services such as employment counseling, and simultaneously addressing polydrug use problems. Finally, in an era of increasing availability of digitized health-related records, our study provides a framework for disease outcome prediction using high dimensional phenotypic data collected via a research instrument.

\section{Abbreviations}

AA: African American

AIC: Akaike information criterion

AUC: area under the curve

CUD: cocaine use disorder

DNN: deep neural network

DSM-5: Diagnostic and Statistical Manual of Mental Disorders, 5th Edition

EA: European ancestry

FTND: Fagerstrom Test for Nicotine Dependence

LASSO: least absolute shrinkage and selection operator

MRI: magnetic resonance imaging

OR: odds ratio

OCD: obsessive-compulsive disorder

OUD: opioid use disorder

PTSD: post-traumatic stress disorder

RF: random forest

SD: standard deviation

SSADDA: Semi-Structured Assessment for Drug Dependence and Alcoholism

SUD: substance use disorder

SVM: support vector machine 


\section{Supplementary materials}

The supplementary materials for this article are available at: https://www.explorationpub.com/uploads/ Article/file/10013_sup_1.pdf.

\section{Declarations}

Acknowledgments

We appreciate the work of recruitment and assessment by James Poling, PhD, at Yale University School of Medicine and the APT Foundation; by Roger Weiss, MD, at McLean Hospital; by Kathleen Brady, MD/PhD and Raymond Anton, MD, at the Medical University of South Carolina; and David Oslin, MD at the University of Pennsylvania. We thank John Farrell, PhD, Section of Biomedical Genetics, Boston University School of Medicine, who provided database management assistance.

\section{Author contributions}

JC designed, conducted the study and wrote the manuscript, R Sherva, KL, R Saitz, MK and LF supervised the study, HK and JG provided the access to the dataset. All authors contributed to manuscript revisions.

\section{Conflicts of interest}

Dr. Kranzler has been a consultant or advisory board member for Indivior and Lundbeck. He is also a member of the American Society of Clinical Psychopharmacology's Alcohol Clinical Trials Initiative, which was supported for the last 3 years by AbbVie, Alkermes, Ethypharm, Indivior, Lilly, Lundbeck, Otsuka, Pfizer, Arbor, and Amygdala Neurosciences.

\section{Ethical approval}

This study was approved by the Institutional Review Boards of Boston University, Yale University and the University of Pennsylvania.

\section{Consent to participate}

Informed consent to participate in the study was obtained from all participants.

\section{Consent to publication}

Not applicable.

\section{Availability of data and materials}

The code used for this analysis can be found at https://github.com/wusixer/feature-selection-using-LASSOSVM-and-Random-Forest and https://github.com/wusixer/feature_selection_in_neural_network.

\section{Funding}

This study was supported by National Institutes of Health grants RC2 DA028909, R01 DA12690, R01 DA12849, R01 DA18432, R01 AA11330, R01 AA017535, 2P50-AA012870, VA Connecticut Healthcare Center, Philadelphia VA MIRECCS, and National Center for Post Traumatic Stress Disorder. The funders had no role in study design, data collection and analysis, decision to publish, or preparation of the manuscript.

\section{Copyright}

(c) The Author(s) 2020.

\section{References}

1. Volkow ND, Frieden TR, Hyde PS, Cha SS. Medication-assisted therapies-tackling the opioid-overdose epidemic. N Engl J Med. 2014;370:2063-6. 
2. Centers for Disease Control and Prevention. Overview of the drug overdose epidemic: behind the numbers. Available from: https://www.cdc.gov/drugoverdose/data/index.html. [Last accessed on 28 May 2019].

3. Acion L, Kelmansky D, van der Laan M, Sahker E, Jones D, Arndt S. Use of a machine learning framework to predict substance use disorder treatment success. PLoS One. 2017;12:e0175383.

4. Che Z, St Sauver J, Liu H, Liu Y. Deep learning solutions for classifying patients on opioid use. AMIA Annu Symp Proc. 2018;2017:525-34.

5. American Psychiatric Association. Diagnostic and statistical manual of mental disorders. 5th ed. Washington, DC: American Psychiatric Pub; 2013.

6. James K, Jordan A. The opioid crisis in Black communities. J Law Med Ethics. 2018;46:404-21.

7. Hansen H, Siegel C, Wanderling J, DiRocco D. Buprenorphine and methadone treatment for opioid dependence by income, ethnicity and race of neighborhoods in New York City. Drug Alcohol Depend. 2016;164:14-21.

8. Om A. The opioid crisis in black and white: the role of race in our nation's recent drug epidemic. J Public Health. 2018;40:e614-5.

9. Santoro TN, Santoro JD. Racial bias in the us opioid epidemic: a review of the history of systemic bias and implications for care. Cureus. 2018;10:e3733.

10. Hellman M. Opioids, opioids, opioids: the plague among middle-aged white Americans. Nordic studies on alcohol and drugs. 2018;35:325-8.

11. Badiani A, Spagnolo PA. Role of environmental factors in cocaine addiction. Curr Pharm Des. 2013;19:6996-7008.

12. Ellis RJ, Wang Z, Genes N, Ma'ayan A. Predicting opioid dependence from electronic health records with machine learning. BioData Min. 2019;12:3.

13. Li X, Chaovalitwongse WA, Curran G, Tilford JM, Felix H, Martin BC. Using machine learning to predict opioid overdoses among prescription opioid users. Value in Health. 2018;21 Suppl 1:S245.

14. Dowell D, Haegerich TM, Chou R. CDC guideline for prescribing opioids for chronic pain-United States, 2016. MMWR Recomm Rep. 2016;65:1-49.

15. Ainscough TS, Brose LS, Strang J, McNeill A. Contingency management for tobacco smoking during opioid addiction treatment: a randomised pilot study. BMJ Open. 2017;7:e017467.

16. Samet JH, Kertesz SG. Suggested paths to fixing the opioid crisis: directions and misdirections. JAMA Netw Open. 2018;1:e180218.

17. Pierucci-Lagha A, Gelernter J, Chan G, Arias A, Cubells JF, Farrer L, et al. Reliability of DSM-IV diagnostic criteria using the semi-structured assessment for drug dependence and alcoholism (SSADDA). Drug Alcohol Depend. 2007;91:85-90.

18. Montalvo-Ortiz JL, Cheng Z, Kranzler HR, Zhang H, Gelernter J. Genomewide study of epigenetic biomarkers of opioid dependence in European- American women. Sci Rep. 2019;9:4660.

19. Wetherill L, Agrawal A, Kapoor M, Bertelsen S, Bierut LJ, Brooks A, et al. Association of substance dependence phenotypes in the COGA sample. Addict Biol. 2015;20:617-27.

20. Bzdok D, Altman N, Krzywinski M. Statistics versus machine learning. Nat Methods. 2018;15:233-4.

21. Beam AL, Kohane IS. Big data and machine learning in health care. JAMA. 2018;319:1317-8.

22. Wong A, Young AT, Liang AS, Gonzales R, Douglas VC, Hadley D. Development and validation of an electronic health record-based machine learning model to estimate delirium risk in newly hospitalized patients without known cognitive impairment. JAMA Netw Open. 2018;1:e181018.

23. Zhang H, Zheng Y, Yoon G, Zhang Z, Gao T, Joyce B, et al. Regularized estimation in sparse highdimensional multivariate regression, with application to a DNA methylation study. Stat Appl Genet Mol Biol. 2017;16:159-71. 
24. Rondina JM, Hahn T, de Oliveira L, Marquand AF, Dresler T, Leitner T, et al. SCoRS--a method based on stability for feature selection and mapping inneuroimaging [corrected]. IEEE Trans Med Imaging. 2014;33:85-98.

25. Zhang X, Lu X, Shi Q Xu XQ, Leung HC, Harris LN, et al. Recursive SVM feature selection and sample classification for mass-spectrometry and microarray data. BMC Bioinformatics. 2006;7:197.

26. Darst BF, Malecki KC, Engelman CD. Using recursive feature elimination in random forest to account for correlated variables in high dimensional data. BMC Genet. 2018;19 Suppl 1:65.

27. Pinaya $\mathrm{WH}$, Gadelha A, Doyle OM, Noto C, Zugman A, Cordeiro $Q$, et al. Using deep belief network modelling to characterize differences in brain morphometry in schizophrenia. Sci Rep. 2016;6:38897.

28. Gelernter J, Panhuysen C, Weiss R, Brady K, Hesselbrock V, Rounsaville B, et al. Genomewide linkage scan for cocaine dependence and related traits: significant linkages for a cocaine-related trait and cocaineinduced paranoia. Am J Med Genet B Neuropsychiatr Genet. 2005;136B:45-52.

29. Sherva R, Wang Q Kranzler H, Zhao H, Koesterer R, Herman A, et al. Genome-wide association study of cannabis dependence severity, novel risk variants, and shared genetic risks. JAMA Psychiatry. 2016;73:472-80.

30. Malison RT, Kalayasiri R, Sanichwankul K, Sughondhabirom A, Mutirangura A, Pittman B, et al. Interrater reliability and concurrent validity of DSM-IV opioid dependence in a Hmong isolate using the Thai version of the Semi-Structured Assessment for Drug Dependence and Alcoholism (SSADDA). Addict Behav. 2011;36:156-60.

31. Hebiri M, Lederer J. How correlations influence Lasso prediction. IEEE Trans Inf Theory. 2013;3:1846-54.

32. Hsu Cw, Chang Cc, Lin Cj. A practical guide to support vector classification. Available from: https://www. csie.ntu.edu.tw/ cjlin/papers/guide/guide.pdf. [Last accessed on 12 Feb 2019]. .

33. Oshiro TM, Perez PS, Baranauskas JA. How many trees in a random forest? In: Perner P, editor. Machine learning and data mining in pattern recognition. MLDM 2012: Proceedings of the 8th International Workshop on Machine Learning and Data Mining in Pattern Recognition; 2012 Jul 13-20; Berlin, Germany. Berlin: Springer; 2012. pp. 154-68.

34. Roy D, Murty KSR, Mohan CK. Feature selection using Deep Neural Networks. In: IJCNN 2015: Proceedings of 2015 International Joint Conference on Neural Networks; 2015 Jul 12-17; Killarney, Ireland. Red Hook: IEEE; 2015. pp. 1-6.

35. Halligan S, Altman DG, Mallett S. Disadvantages of using the area under the receiver operating characteristic curve to assess imaging tests: a discussion and proposal for an alternative approach. Eur Radiol. 2015;25:932-9.

36. Bekkar M, Djemaa HK, Alitouche TA. Evaluation measures for models assessment over imbalanced data sets. J Inf Eng Appl. 2013;3:27-38.

37. Weng WH, Wagholikar KB, McCray AT, Szolovits P, Chueh HC. Medical subdomain classification of clinical notes using a machine learning-based natural language processing approach. BMC Med Inform Decis Mak. 2017;17:155.

38. Saito T, Rehmsmeier M. Precrec: fast and accurate precision-recall and ROC curve calculations in R. Bioinformatics. 2017;33:145-7.

39. Pedregosa F, Varoquaux G, Gramfort A, Michel V, Thirion B, Grisel O, et al. Scikit-learn: Machine Learning in Python. J Mach Learn Res. 2011;12:2825-30.

40. Gowin JL, Ball TM, Wittmann M, Tapert SF, Paulus MP. Individualized relapse prediction: personality measures and striatal and insular activity during reward-processing robustly predict relapse. Drug Alcohol Depend. 2015;152:93-101.

41. Erguzel TT, Noyan CO, Eryilmaz G, Ünsalver BÖ, Cebi M, Tas C, et al. Binomial logistic regression and artificial neural network methods to classify opioid-dependent subjects and control group using quantitative EEG power measures. Clin EEG Neurosci. 2019;50:303-10. 
42. Ahn WY, Vassileva J. Machine-learning identifies substance-specific behavioral markers for opiate and stimulant dependence. Drug Alcohol Depend. 2016;161:247-57.

43. Ahn WY, Ramesh D, Moeller FG, Vassileva J. Utility of machine-learning approaches to identify behavioral markers for substance use disorders: impulsivity dimensions as predictors of current cocaine dependence. Front Psychiatry. 2016;7:34.

44. Buu A, Johnson NJ, Li R, Tan X. New variable selection methods for zero-inflated count data with applications to the substance abuse field. Stat Med. 2011;30:2326-40.

45. Robins LN. The intimate connection between antisocial personality and substance abuse. Soc Psychiatry Psychiatr Epidemiol. 1998;33:393-9.

46. Brook JS, Whiteman M, Finch SJ, Cohen P. Young adult drug use and delinquency: childhood antecedents and adolescent mediators. J Am Acad Child Adolesc Psychiatry. 1996;35:1584-92.

47. Dragisic T, Dickov A, Dickov V, Mijatovic V. Drug addiction as risk for suicide attempts. Mater Sociomed. 2015;27:188-91.

48. Phillips J, Carpenter KM, Nunes EV. Suicide risk in depressed methadone-maintained patients: associations with clinical and demographic characteristics. Am J Addict. 2004;13:327-32.

49. Harned MS, Najavits LM, Weiss RD. Self-harm and suicidal behavior in women with comorbid PTSD and substance dependence. Am J Addict. 2006;15:392-5.

50. Trémeau F, Darreye A, Staner L, Corrêa H, Weibel H, Khidichian F, et al. Suicidality in opioid-dependent subjects. Am J Addict. 2008;17:187-94.

51. Oliveto AH, Feingold A, Schottenfeld R, Jatlow P, Kosten TR. Desipramine in opioid-dependent cocaine abusers maintained on buprenorphine vs methadone. Arch Gen Psychiatry. 1999;56:812-20.

52. Krantz MJ, Mehler PS. Treating opioid dependence: growing implications for primary care. Arch Intern Med. 2004;164:277-88.

53. Schottenfeld RS, Pakes J, Ziedonis D, Kosten TR. Buprenorphine: dose-related effects on cocaine and opioid use in cocaine-abusing opioid-dependent humans. Biol Psychiatry. 1993;34:66-74.

54. Mannelli P, Wu LT, Peindl KS, Gorelick DA. Smoking and opioid detoxification: behavioral changes and response to treatment. Nicotine Tob Res. 2013;15:1705-13.

55. Thylstrup B, Schrøder S, Hesse M. Psycho-education for substance use and antisocial personality disorder: a randomized trial. BMC Psychiatry. 2015;15:283.

56. Socías ME, Wood E, Lake S, Nolan S, Fairbairn N, Hayashi K, et al. High-intensity cannabis use is associated with retention in opioid agonist treatment: a longitudinal analysis. Addiction. 2018;113:2250-8.

57. Wiese B, Wilson-Poe AR. Emerging evidence for cannabis' role in opioid use disorder. Cannabis Cannabinoid Research. 2018;3:179-89.

58. Humphreys K, Saitz R. Should physicians recommend replacing opioids with cannabis? Jama. 2019;321:639-40.

59. Carpenter CS. Workplace drug testing and worker drug use. Health Serv Res. 2007;42:795-810.

60. Kosten TR, George TP. The neurobiology of opioid dependence: implications for treatment. Sci Pract Perspect. 2002;1:13-20.

61. Barry DT, Steinberg MA, Wu R, Potenza MN. Characteristics of black and white callers to a gambling helpline. Psychiatr Serv. 2008;59:1347-50.

62. Welte JW, Barnes GM, Tidwell MC, Hoffman JH, Wieczorek WF. Gambling and problem gambling in the United States: changes between 1999 and 2013. J Gambl Stud. 2015;31:695-715.

63. Rounsaville BJ, Kleber HD. Untreated opiate addicts: how do they differ from those seeking treatment? Arch Gen Psychiatry. 1985;42:1072-7.

64. Johnson RE, Chutuape MA, Strain EC, Walsh SL, Stitzer ML, Bigelow GE. A comparison of levomethadyl acetate, buprenorphine, and methadone for opioid dependence. N Engl J Med. 2000;343:1290-7. 
65. Fiellin DA, Weiss L, Botsko M, Egan JE, Altice FL, Bazerman LB, et al. Drug treatment outcomes among HIV-infected opioid-dependent patients receiving buprenorphine/naloxone. J Acquir Immune Defic Syndr. 2011;56 Suppl 1:S33-8.

66. Kazakova OB, Tret'iakova EV, Kukovinets OS, Tolstikov GA, Nazyrov TI, Chudov IV, et al. Synthesis and pharmacological activity of amides and ozonolysis product of maleopimaric acid. Bioorg Khim. 2010;36:832-40. Russian.

67. Liang W, Chikritzhs T. Reduction in alcohol consumption and health status. Addiction. 2011;106:75-81.

68. Wells EA, Morrison DM, Gillmore MR, Catalano RF, Iritani B, Hawkins JD. Race differences in antisocial behaviors and attitudes and early initiation of substance use. J Drug Educ. 1992;22:115-30.

69. MacDonald GJ. Atheist gatherings are mostly white, male. Available from: https://www.mysanantonio. com/news/religion/article/Atheist-gatherings-are-mostly-white-male-944612.php. [Last accessed on 11 Sep 2019].

70. Roberts AL, Gilman SE, Breslau J, Breslau N, Koenen KC. Race/ethnic differences in exposure to traumatic events, development of post-traumatic stress disorder, and treatment-seeking for post-traumatic stress disorder in the United States. Psychol Med. 2011;41:71-83.

71. Moscati A, Mezuk B. Losing faith and finding religion: religiosity over the life course and substance use and abuse. Drug Alcohol Depend. 2014;136:127-34.

72. Smyth BP, Barry J, Keenan E, Ducray K. Lapse and relapse following inpatient treatment of opiate dependence. Ir Med J. 2010;103:176-9.

73. Scherbaum N, Specka M. Factors influencing the course of opiate addiction. Int J Methods Psychiatr Res. 2008; 17 Suppl 1:S39-44.

74. Cho J, Lee K, Shin E, Choy G, Do S. How much data is needed to train a medical image deep learning system to achieve necessary high accuracy? arXiv 1511.06348 [Preprint]. 2016 [cited 31 Mar 2019]. Available from: https://arxiv.org/abs/1511.06348.

75. Shiraly R, Taghva M. Factors associated with sustained remission among chronic opioid users. Addict Health. 2018;10:86-94.

76. Haukoos JS, Newgard CD. Advanced statistics: missing data in clinical research-part 1: an introduction and conceptual framework. Acad Emerg Med. 2007;14:662-8. 